\title{
Slow extinction of conditioned responding following exposure to two bouts of massed shock
}

\author{
DOUGLAS A. WILLIAMS \\ University of Winnipeg, Winnipeg, Manitoba, Canada
}

\begin{abstract}
Extinction of rats' conditioned defensive freezing responses in a context associated with two bouts of massed shock ( $3 \mathrm{sec}$ ) separated by a long unreinforced interbout interval was slower than that in a context associated with distributed shock $(60 \mathrm{sec})$. Resistance to extinction following two bouts of massed shock depended on the rats' remaining undisturbed in the conditioning context during the long unreinforced interbout interval. Slow extinction of freezing was attributed to either the summation of temporal conditioning at the early and late session times or the formation of an association between the early and late bouts of shock. Importantly, the effects of the two bouts of massed shock could not be explained by what is known about the reinforcing effectiveness of massed shock.
\end{abstract}

When a biologically relevant unconditioned stimulus (US) is used as a reinforcing agent in Pavlovian conditioning experiments, it necessarily occurs in a particular context and at specific times. Although the role of context in learning and performance has been widely investigated (e.g., Balsam \& Tomie, 1985), much less attention has been paid to whether animals are sensitive to regularities in the temporal distribution of USs during conditioning. One of the best examples of sensitivity to US-US temporal relations was reported by Goddard and Jenkins (1988), who showed that pigeons could learn the arrival times of successive USs scheduled at fixed intervals. This learning was shown by the ability of excitatory temporal cues to block conditioning of a discrete keylight CS that was later introduced as a redundant signal for the US.

Another way to show that temporal factors have an important role in Pavlovian conditioning is to examine different US distributions that have the same nominal density of reinforcement. In the experiments reported in this article, I demonstrate how the simplest of conditioned responses (CRs) can be profoundly influenced by the temporal distribution of USs within conditioning sessions. When rats are placed in an environment that is associated with unsignaled footshock, they become frightened and freeze (e.g., Blanchard \& Blanchard, 1969). The magnitude of defensive freezing is determined by what has been learned about the shock US. If conditioned freezing depends on the mere number of context-US pairings, and not temporal factors, the level of freezing upon reexposure to the context and the rate of extinction of the freezing response should be virtually identical in groups matched

This research was supported by a grant from the Natural Sciences and Engineering Research Council of Canada. I thank Elena Tinio, Kerry Sagness, and Orëlund Pennell for their assistance in conducting this research, and Vin LoLordo for comments on a draft of this article. Requests for reprints should be addressed to $D$. Williams, Department of Psychology, University of Winnipeg, Winnipeg, MB, Canada R3B 2E9 (e-mail: williams@uwpg02.uwinnipeg.ca). for number of shocks, time of the first and last shocks, and session duration. On the other hand, if rats are sensitive to temporal relations among shocks, large differences in conditioned responding should emerge as a function of when USs occur in the session.

The study of temporal conditioning by using this approach is complicated by the potential contribution of nonassociative factors to US processing. For example, some types of US distributions may favor sensitization, and others may favor habituation. If so, differences in conditioned freezing after exposure to different US distributions could be due to nonassociative processes and not to temporal conditioning. One way to rule out nonassociative factors would be to arrange US presentations in such a way that nonassociative effects oppose temporal conditioning. In the present experiments, this opposition of effects was accomplished by presenting two bouts of massed shocks ( 5 shocks per bout, separated by 3-sec intervals) in a single conditioning session that was separated by a long unreinforced interval (split procedure). This arrangement of USs was compared with a distributed procedure, in which 10 shocks were spaced at fixed 60-sec intershock intervals. Freezing in the split and distributed groups was assessed in the conditioning context in the absence of shock in the extinction test that followed.

From a temporal conditioning perspective, it would not be surprising to observe slower extinction of conditioned responding in the split procedure than in the distributed procedure. Slow extinction would be expected on several bases. First, rats may learn that independent bouts of shock occur early and late in the conditioning session. A sensitivity to the time of bout presentation would encourage freezing throughout the test session. Second, rats receiving the split regimen may learn that an early bout of shock is followed much later by another bout of shock. This bout-to-bout signaling also could impede extinction. On the other hand, the absence of the US should be readily discriminated in the distributed group during testing be- 
cause of the repeated failure of the US to occur at its designated 60-sec intershock interval.

Extinction of conditioned responding that is slower in a split group than in a distributed group is unlikely to have a nonassociative basis. Fanselow and Tighe (1988) demonstrated that when shocks are massed at very brief 3-sec intervals, there is a rather profound deficit in defensive freezing upon reexposure to the shocked environment; they found that a 3-sec intershock interval supports much lower levels of defensive freezing than does either a 30or 60 -sec intershock interval. Indeed, the level of conditioning reported at $3 \mathrm{sec}$ was not all that different from that produced by a single shock. This phenomenon, the massed-shock deficit, certainly should not encourage slow extinction of conditioned freezing in the split group. Massed shock is clearly a less effective reinforcing agent than is distributed shock, and minimal levels of contextual conditioning should be observed in the split procedure.

Consistent with the temporal conditioning hypothesis, Experiment 1 demonstrated that two separated bouts of massed shock led to greater resistance to extinction than the same number of distributed shocks. In subsequent experiments I explored the mechanisms underlying enhanced freezing following two bouts of massed shock. Several accounts were ruled out, including (1) opponent-process theory, (2) an account holding that freezing misrepresents fear level because it is a duration measure, (3) sensitization of the freezing response by prior massed shock, (4) partial reinforcement of contextual cues, and (5) chronic fear caused by unpredictable shock. The only surviving account was one suggesting that freezing was evoked by a temporal anticipation of shock.

\section{EXPERIMENT 1}

In Experiment 1, conditioned freezing was examined under the five conditioning procedures illustrated in Figure 1 . In the split procedure, two bouts of five massed shocks separated by a long 516-sec interbout interval were presented in a single session of conditioning. This condition was contrasted with a distributed procedure, in which shocks were separated by fixed 60 -sec intervals. The two groups had identical shock densities; the first and last shocks occurred at exactly the same times and differed only in the spacing of the intervening shocks. If the known effectiveness of massed and distributed shock is the only factor determining freezing, then the split 3-sec procedure should yield little conditioned responding and certainly less responding than the distributed $60-\mathrm{sec}$ procedure. Similar or higher levels of freezing in the split group than in the distributed group would indicate that temporal factors had compensated for the lack of effectiveness of massed shock.

Three control groups were included in order to further assess the viability of the temporal conditioning hypothesis. Two massed control groups were included to confirm that massed shock supports less conditioned freezing than distributed shock. Shock number was controlled by presenting a single bout of 10 massed shocks, either early or late in the conditioning session. Fanselow and Tighe (1988) reported massed-shock deficits with $2,3,9$, and 16 shocks. Thus, there was good reason to expect that 10 shocks would produce a massed-shock deficit. A third control group received only the first shock of each bout of the split group. This "split/first" group allowed an assessment of whether single shocks presented early and late in the conditioning session could substitute for the bouts of 5 shocks in the split group. If the 4 added shocks of each bout of the split group were effective, then the split group should freeze more than the split/first group.

In addition to testing the temporal conditioning hypothesis, another aim of Experiment 1 was to provide insight into freezing as an index of conditioned fear. Freezing can be viewed as a rather peculiar defensive $C R$ in that it occurs at very low levels of conditioned fear. It is noteworthy in this regard that freezing, a preparatory re-

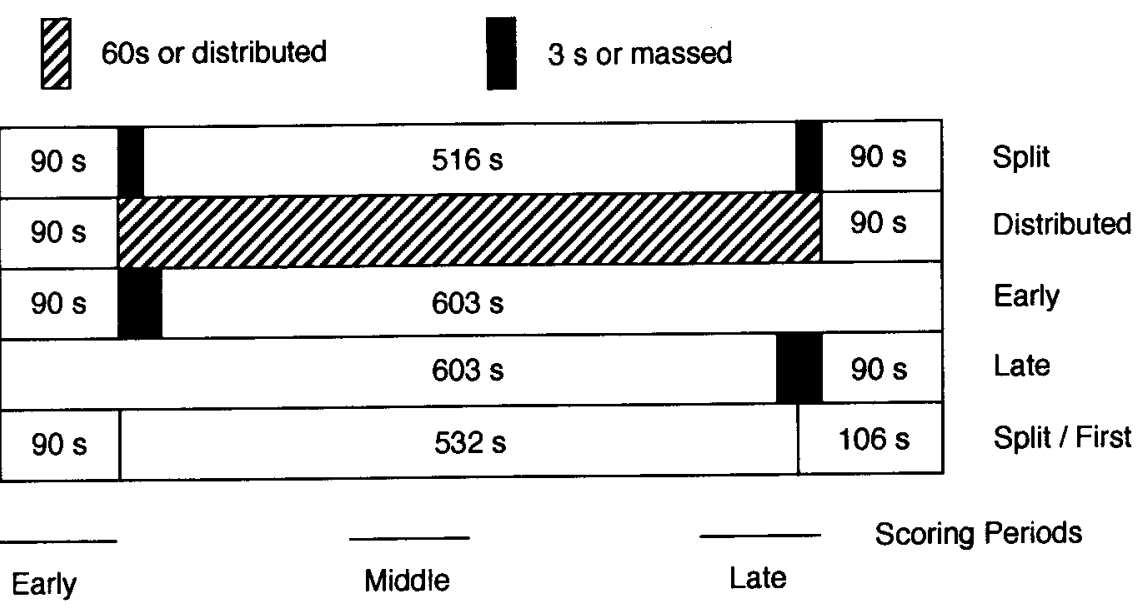

Figure 1. The temporal arrangement of shock USs for the various conditioning procedures used in Experiment 1. 
sponse, is measured in terms of its duration (i.e., the percentage of sampling periods in which it occurs), and not its intensity. In Experiment 1, shocks in the split procedure were scheduled to occur throughout the session. This arrangement could engender low levels of fear for long periods of time, producing a high percentage of time samples that included a freezing response. If this "lowintensity freezing" explanation has merit, similar levels of freezing should be observed in the split, split/first, and distributed groups simply by virtue of matching the times of the first and last shocks.

\section{Method}

Subjects. The subjects were 55 naive, male Sprague-Dawley rats obtained from Charles River, St. Constant, Quebec. The rats weighed between 250 and $295 \mathrm{~g}$ and were 90 days old upon arrival. They were housed individually in wire-mesh cages in a colony that was maintained on a 12:12-h light:dark cycle, and they had free access to food and water. The experiment was conducted in the light portion of the cycle. The rats arrived about 3 weeks prior to the beginning of the experiment and were handled on alternate days.

Apparatus. The conditioning apparatus was a standard operant chamber, measuring $30 \times 30 \times 27.5 \mathrm{~cm}$, with clear acrylic sides and ceiling. The front and back panels were aluminum. The front panel contained a recessed food tray in the center, just above the grid floor, and jeweled lights on either side midway between the floor and ceiling; neither the food tray nor the lights were used. The front panel also contained a lever that was inoperable. The floor consisted of 6-mm stainless steel rods spaced $1.6 \mathrm{~cm}$ apart center to center. The floor could be electrified by a constant-current shocker and scrambler (Coulbourn Instruments). This apparatus was placed in an open, sound-attenuated cube so that the rat's behavior could be videotaped. The overhead room lights were extinguished. A $40-\mathrm{W}, 120-\mathrm{V}$ ac lamp located $2 \mathrm{~m}$ from the apparatus bathed the operant chamber in red light, which provided ample illumination for video recording. A Panasonic BL204 video camera was positioned about $1.5 \mathrm{~m}$ away from the apparatus. An Apple II computer controlled the presentation and timing of shocks.

Procedure. On Day 1, the rats were given the opportunity to explore the to-be-conditioned context for $730 \mathrm{sec}$ in the absence of shock. Experimental manipulations occurred on a single day in the next conditioning session. The rats were randomly assigned to one of five groups $(n=11)$. Four of the five groups received 10 shocks $(0.5 \mathrm{~mA}, 1 \mathrm{sec})$. In the distributed group, the 10 shocks were spaced $60 \mathrm{sec}$ apart. The first shock occurred $90 \mathrm{sec}$ after placement in the conditioning environment, and the last shock terminated $90 \mathrm{sec}$ prior to the end of the session. In the split group, two bouts of 5 massed shocks were presented; the first shock of Bout 1 occurred $90 \mathrm{sec}$ after context placement, and the last shock of Bout $2 \mathrm{oc}$ curred $90 \mathrm{sec}$ prior to the end of the session. The 5 shocks of each bout were spaced $3 \mathrm{sec}$ apart. These parameters produced a 516-sec rest period between the 5th and 6th shocks when combined with the 730-sec session length used throughout the experiment. Two other groups also received massed shock. In the early group, a single bout of 10 massed shocks began $90 \mathrm{sec}$ after placement. The rats remained in the conditioning chamber for an additional $613 \mathrm{sec}$ following the 10th shock, in order to equate the groups for session length. The rats in the late group, in contrast, received a bout of 10 shocks at the end of the conditioning session. The first shock occurred $613 \mathrm{sec}$ after they were placed in the conditioning chamber; the 10th shock terminated $90 \mathrm{sec}$ before the end of the session. Also included was a group that received only the first shock of each bout of the split procedure. This split/first group received Shocks 1 and 6 . The other 8 shocks of the split procedure were deleted.
These conditioning manipulations were followed by 3 days of freezing tests conducted in the absence of shock. The rats were placed in the conditioning chamber and freezing was assessed early $(0-90 \mathrm{sec})$, midway $(275-365 \mathrm{sec})$, and late $(550-640 \mathrm{sec})$ in the session. The test sessions lasted $730 \mathrm{sec}$. A time-sampling technique was used to measure freezing, which was scored 18 times during each of the $90-\mathrm{sec}$ recording periods. Once every $5 \mathrm{sec}$, the rats were scored as either freezing or not. Freezing was scored only at the moment of a brief recording signal and was defined by the absence of visible movement, except that necessitated by respiration. The primary observer, whose data are reported in this article, was aware of group membership but was blind to the experimental hypothesis. Test sessions were videotaped to allow independent rescoring by a second observer, who was uninformed about group membership. An auditory signal, superimposed on the videotapes, paced the scoring. Interobserver reliability was calculated by correlating the total number of freezing responses scored by each observer on 198 randomly selected recording periods. Reliability was uniformly high when averaged across each of the experiments $(r=.96)$.

Statistical analysis. The percentage of intervals in which freezing was observed was analyzed by using an analysis of variance (ANOVA). Because group differences were expected to vary as freezing extinguished over the test, the simple effect of group was examined separately at each of the nine test intervals. Error rates were controlled on a per contrast basis by using tables provided by Rodger (1975). Tests of simple effects used the pooled ANOVA error; degrees of freedom were calculated from Satterthwaite's (1946) approximation. If the simple effect of group was significant at a particular interval, group orderings were determined post hoc by using the procedures developed by Rodger (1974). This involved choosing a set of mutually orthogonal contrasts and evaluating them against Rodger's (1975) special values of $F[E \alpha]$. Type I error rates were controlled by ensuring that the proportion of all true nulls rejected in error did not exceed $E \alpha=.05$.

\section{Results and Discussion}

The mean percentage of samples scored as freezing in the early $(E)$, middle $(M)$, and late $(L)$ periods of the 3-day extinction test are shown in Figure 2. The major finding was that separating massed shocks into two bouts slowed extinction of freezing. Although the split group froze at modest levels on initial exposure to the context, freezing did not diminish quickly in this group over the 3-day test. This enhancement of freezing in the split group was supported by an ANOVA, which revealed significant group differences in the early $[F(4,138)=4.28$; distributed $>$ split $=$ early $=$ split/first $>$ late $]$, middle $[F(4,138)=$ 3.80; split $=$ distributed $>$ early $=$ split $/$ first $>$ late $]$, and late $[F(4,138)=3.10$; split $>$ late $=$ split $/$ first $=$ distributed $>$ early] intervals of Day 1 . Reliable group differences were also observed in the early intervals of Day $2[F(4,138)=3.09$; split $>$ distributed $=$ early $>$ split/first $=$ late $]$ and Day $3[F(4,138)=2.20$; split $>$ early $=$ distributed $>$ split $/$ first $=$ late]. The simple effect of group in the middle and late periods of Day 2 was not statistically significant; the variance among the five means was considerably smaller in these periods even though some pairwise differences were greatest at this point of the experiment.

Especially surprising from a nonassociative view was the observation that, on some occasions, freezing in the split group was significantly more vigorous than in the dis- 


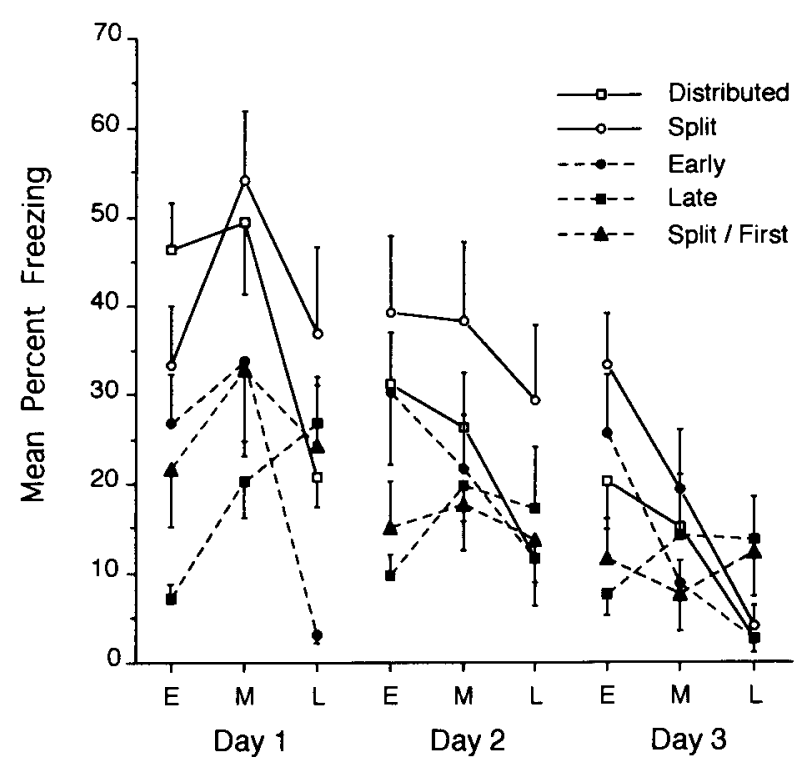

Figure 2. Freezing during the 3-day test of Experiment 1 as a function of group (distributed, split, early, late, and split/first) during the three daily observation intervals ( $E=$ early, or 0-90 sec; $M=$ middle, or 275-365 sec; and $L=$ late, or 550-640 sec).

tributed group. Freezing was significantly higher in the split group than in the distributed group in the late period of Day 1 and during the early intervals of Days 2 and 3. Thus, not only was more freezing consistently observed in the split group than in the early, late, and split/first groups, but also there was more freezing in the split group than in the distributed group later in the test. Although freezing was slow to extinguish in the split group, the final level of conditioning may not have been as high as that in the distributed group. During the first observation interval on Day 1 of testing, freezing was significantly higher in the distributed group than in the split group. The different within-session extinction profiles of these groups strongly implicates a role for temporal factors in this simple conditioning preparation. This conclusion follows, because the rate of extinction could not be predicted by the initial level of freezing on exposure to the context, thus implying that something in addition to a context-US association was learned.

As mentioned earlier, Figure 2 shows more freezing in the split group than in either the early or late massedshock groups. This difference was most evident in the middle and late recording periods on Day 1 . The early and late groups, although showing similar overall levels of freezing, froze selectively at the conditioning time. The early group froze at the beginning of the session, and freezing declined as the session continued. The late group did not freeze at high levels upon placement in the context, but did so increasingly as the session progressed. These results show the sensitivity of the early and late groups to the temporal locus of the bout of shocks.

A surprising amount of freezing was observed in the split/first group on Day 1: The split/first group froze more than did the late group at the beginning of the session and more than did the early group at the end of the session. Thus, there may be some truth to the conjecture that scheduling shocks at the beginning and end of the session may evoke freezing throughout the session. However, this effect was not substantial enough to explain the slow extinction of freezing in the split group. The split/ first group froze at lower levels than did the split group. Freezing rapidly extinguished in the split/first group on Days 2 and 3.

The results of Experiment 1 provide important new information about the conditions under which massed shock serves as an effective reinforcer, and they suggest that a temporal conditioning mechanism underlies the enhancement of conditioning. Fanselow and Tighe (1988) interpreted findings from several experiments on the massedshock deficit in terms of an opponent-process mechanism (Solomon \& Corbit, 1974). They suggested that massed USs may interfere with contextual conditioning because shock, a motivationally significant event, elicits an initial burst of activity that is followed by an unconditioned counterreaction. If the shocks are too closely spaced, then the shock on Trial $\mathrm{N}+1$ occurs during the counterreaction evoked by the previous shock on Trial $\mathrm{N}$. This condition results in a substantial loss of US effectiveness and explains why a single bout of massed USs produces similar levels of conditioning as a single shock: Only the first shock of a bout of massed shocks is fully effective.

The results of Experiment 1 can be reconciled with this opponent-process theory if it is assumed that the counterreaction to shock dissipates only slightly over the postshock interval between 3 and $60 \mathrm{sec}$, but then degrades rapidly thereafter. These conditions would create two maximally effective shocks in the split procedure-the first and sixth shocks. In the distributed group, all the shocks except Shock 1 occurred within the 60 -sec temporal window defining the hypothesized postshock counterreaction, and thus their effectiveness would have been diminished. Nonetheless, these putative changes in the temporal dynamics of the counterreaction are inconsistent with the brief intervals that are known to produce the massed-shock deficit (i.e., $16 \mathrm{sec}$ or less; Fanselow \& Tighe, 1988; Williams, Frame, \& LoLordo, 1991) and cannot explain why the split/first group also did not freeze more than did the distributed group. Thus, the magnitude of freezing evoked by different schedules of shock presentation cannot be reduced to the summation of two counteracting states elicited unconditionally by the US.

Recent experimentation in Fanselow's laboratory (Fanselow, DeCola, \& Young, 1993) also has raised doubts about the original opponent-process interpretation of the massed-shock deficit. Contrary to the predictions of opponent-process theory, they reported that the massedshock deficit was not enhanced by shock preexposure in another context or attenuated by the opioid antagonist naloxone. These results also allayed the concern (Williams et al., 1991) that freezing may misrepresent the true level of conditioning in a massed group because of a perfor- 
mance artifact. Fanselow et al.'s current view of the massed-shock deficit holds two factors responsible for the poor conditioning. First, massed shock may not allow for adequate processing of the context. This mechanism is of special concern when the first massed shock occurs immediately after placement in the conditioning apparatus. In Experiment 1, the split group, like the other groups, was preexposed to the conditioning context. The first shock of conditioning was presented $90 \mathrm{sec}$ after placement in the context. Although this aspect of the procedure may have increased the effectiveness of massed shock, there is no reason, on this first mechanism, to predict slower extinction of responding in the split group relative to the distributed group: There was less time to process contextual cues during the short 3-sec intershock intervals in the split group than there was in the 60-sec intershock intervals in the distributed group.

Of greater relevance to the present experiments is Fanselow et al.'s (1993) suggestion that association formation is inhibited during the motoric unconditioned activity burst at shock presentation. When an animal is confronted with a painful US, a vigorous behavioral unconditioned response (UR), consisting of locomotion, vocalization, and other primary defensive responses, is elicited. If a second US occurs at the time of the activity burst UR, fear conditioning could be suppressed by the primary defensive system protecting the animal against the aversive US. Two lines of evidence support this mechanism. First, lesions of the brain areas involved in primary defensive responding enhance the acquisition of conditioned freezing (Landeira-Fernandez, Fanselow, \& De Oca, 1992). Second, preexposure to shock in a context different from conditioning can eliminate the massed shock deficit, provided that the preexposure renders the shock less capable of eliciting a vigorous activity burst (e.g., Fanselow et al., 1993). If so, an analogous sensitization effect, and not temporal conditioning, could explain slow extinction in the split group in Experiment 1 if the first bout of massed shock sensitized the rats to the second bout. This sensitization account, however, seems somewhat implausible for the results of Experiment 1, given that the shock parameters during Bout 1 were not especially conducive to sensitization. Massed presentations do not promote a longterm reduction in the activity burst to a shock US (Fanselow et al., 1993). Despite this caveat, caution requires an experimental test of the sensitization account.

\section{EXPERIMENT 2}

In Experiment 2, I tested the hypothesis that separating shocks into two bouts may increase the capacity of massed shock to support the formation of context-US associations. A critical feature of the sensitization account is that preexposure and conditioning need not occur in the same place. Fanselow et al. (1993) found that shock preexposure in Context 1 enhanced subsequent freezing in Context 2 when the latter context was reinforced with massed shock. In their experiment, however, rats were preexposed to 15 widely distributed shocks $(360 \mathrm{sec})$, and the sensitization session was separated from the massed conditioning session by $24 \mathrm{~h}$. These parameters were chosen in order to produce a long-term reduction of the disruptive behavioral effects of shock. Experiment 2 was an evaluation of the sensitization account of the slow extinction of conditioned freezing in the split procedure by using the parameters of Experiment 1.

Groups of rats were exposed to one context, returned to the colony briefly, and then given five massed shocks in the conditioning context. The groups differed in terms of whether the pretreatment context was the same as or different from the conditioning context, and whether five massed shocks were delivered during the preexposure phase. If an initial bout of massed shock sensitizes rats to the US, then the preshocked groups should freeze at higher levels than the unshocked controls when they are later tested in the conditioning context. This outcome should be obtained even when preshocks and conditioning shocks occur in different contexts. Alternatively, if preexposure to massed shocks results in habituation, and thereby reduces subsequent conditionability, then a conditioning context associated with habituated shock should evoke less freezing than one associated with novel shock. Furthermore, rats preshocked in a context different from that used for conditioning should freeze less than the unshocked controls upon reexposure to the conditioning context.

\section{Method}

Subjects and Apparatus. The subjects were 48 male SpragueDawley rats, housed under the conditions employed in Experiment 1. The operant chamber, described in Experiment 1, was used as one context. The other context was a $58 \times 38 \times 18 \mathrm{~cm}$ rectangular choice box that was located on the floor in an open room with illuminated overhead lights. The walls of the box were plywood, and the floor consisted of $2.5-\mathrm{mm}$ stainless steel bars spaced $1.5 \mathrm{~cm}$ apart that could be electrified. The lid was clear acrylic. The box was divided along its length into two equal-sized compartments that were separated by a wooden wall. The walls and floor tray of one compartment were painted white; the other compartment was painted black and was never used. An $18 \times 18 \mathrm{~cm}$ opening in the middle of the wall that divided the chamber was blocked by a transparent acrylic door, which prevented the rats from entering the black compartment from the white compartment. The contexts used in Experiment 2 were counterbalanced as Contexts 1 and 2 .

Procedure. On Day 1, the rats were allowed to explore both contexts in the same sequence and for the same amount of time as in later testing. Context 1 was presented first. The rats explored this context for $200 \mathrm{sec}$ and were then carried back to their wire cages in the colony room. Context 2 , the conditioning context, was then presented for $200 \mathrm{sec}$. The interval between the termination of the first session and placement in the second context was approximately $336 \mathrm{sec}$. This included about $40 \mathrm{sec}$ of transportation time back and forth to the colony room.

Three groups of rats $(n=16)$ were then formed for the next conditioning day. The preshock/diff group experienced a bout of five massed shocks $(0.5 \mathrm{~mA}, 1 \mathrm{sec})$ in Context 1 . After resting briefly in the colony room, they were returned to the conditioning context, Context 2, for another bout of massed shock. Five shocks, spaced $3 \mathrm{sec}$ apart, were presented in each context. The first shock was presented $90 \mathrm{sec}$ after placement, the session terminated $90 \mathrm{sec}$ 
after the last shock, and the interval between context presentations was $336 \mathrm{sec}$. Thus, the conditioning procedure was an analogue of the split procedure used in the previous experiment. With the 90 -sec periods taken into account, the approximate interval between the last shock in Context 1 and the first shock in Context 2 was $516 \mathrm{sec}$, which was the same interbout interval used in Experiment 1 . The rats in the unshocked group were treated identically, except that no shocks were presented in Context 1 . These two groups were contrasted with a preshock/same group that received two bouts of shocks in the same context, Context 2 . Like the other groups, this group briefly remained in the colony room between conditioning sessions, allowing a determination of the effects of two sessions of shock in the same context separated by a brief rest interval. Differences in conditioned freezing were determined the next day in the absence of shock. All the rats were exposed to Contexts 1 and 2 in the same sequential fashion as that during the initial exploration day. Conditioned freezing was measured every $5 \mathrm{sec}$. The two sessions lasted $200 \mathrm{sec}$.

\section{Results and Discussion}

The data of primary interest are the level of freezing in the target context (Context 2). The most important observation was that less freezing was observed in the preshock/ diff group than in the unshocked and preshock/same groups. This difference was statistically significant and can be seen in the right-hand side of Figure $3[F(2,87)=$ 7.16; preshock/same $=$ unshocked $>$ preshock/diff] . Thus, preshocks did not sensitize the rats to the later effects of massed shock; if anything, prior experience with shock caused habituation. This finding is inconsistent with the sensitization account of the enhanced freezing in the split group in Experiment 1. The difference in conditioning between the preshock/diff and preshock/same groups in Context 2 also confirms the discriminability of the contexts. These groups would not have differed if the contexts had not been discriminated, because they received the same total amount of shock.

These results do not necessarily conflict with the finding of Fanselow et al. (1993) that distributed preshock

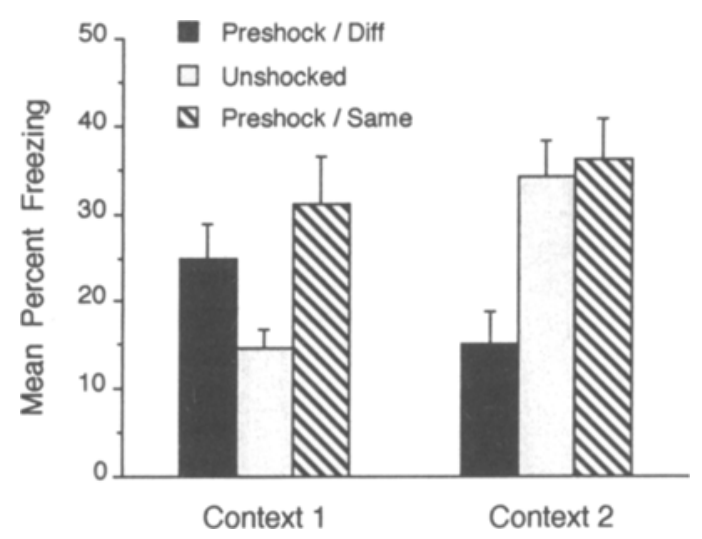

Figure 3. Mean percentage of freezing during testing in Context 1 (left) and Context 2 (right) as a function of preexposure treatment in the context presented first in the conditioning sequence (preshock/diff $=$ one bout massed shock in Context 1 ; unshocked = sit in Context 1; preshock/same = one bout of massed shock in Context 2). can sensitize rats to the later effects of massed shock in another context. In the present experiment, an attempt was made to sensitize the preshock/diff group to massed shock by preexposing them to a prior bout of massed and not distributed shock. Moreover, preshocks in Context 1 were quickly followed by conditioning in Context 2 . The sensitization and conditioning stages in the experiment reported by Fanselow et al. were separated by $24 \mathrm{~h}$.

Figure 3 also shows the mean percentage of observation intervals in which freezing was observed in Context 1. As expected, more freezing was observed in Context 1 in the preshock/diff group than in the unshocked group $[F(2,87)=3.73$; preshock/same $>$ preshock/diff $>$ unshocked]. The only surprising result of Experiment 2 was the high level of freezing in the preshock/same group in Context 1 . The rats in the preshock/same group had never been shocked in Context 1 , but they froze in its presence. There are a number of mechanisms that might explain this odd result. Some possibilities include sensory preconditioning between serially presented contexts (i.e., Stage 1 , Context $1 \rightarrow$ Context 2 ; Stage 2 , Context $2 \rightarrow U S$; and Test, Context $1 \rightarrow$ Context $2 \rightarrow$ US), or the rats' having learned that both sessions were reinforced regardless of the physical surroundings. Whatever the mechanism, the effect does not diminish the demonstration of transsituational habituation in the preshock/same group.

\section{EXPERIMENT 3}

Conditioned responding is not always a good indicator of the strength of an association. Perhaps the best known example of this caveat is the partial reinforcement extinction effect in instrumental conditioning. When instrumental responding is intermittently reinforced, subsequent extinction is surprisingly slow--much slower than when responding is continuously reinforced. The most widely accepted interpretation of slow extinction after partial reinforcement is that responding persists because subjects have learned to respond in the presence of the memory of unreinforced instrumental responses (Capaldi, 1966, 1967). A similar partial reinforcement mechanism might explain why two bouts of massed shock retarded extinction in Experiment 1 . The memory of the long unreinforced interbout interval may have signaled subsequent reinforcement and increased resistance to extinction.

Consider the observation that, in instrumental conditioning, resistance to extinction increases with (1) the number of occasions that nonreinforcement $(N)$ precedes reinforcement (N-R transitions; Capaldi, 1966, 1967) and (2) the length and variability of nonreinforcement $(\mathrm{N}$-length; Capaldi, 1964). Factors such as these might work together to increase resistance to extinction in a split group. For example, a split group with a 516-sec unreinforced interbout interval would experience one highly discriminable $\mathrm{N}-\mathrm{R}$ transition following exposure to shock. On the other hand, the close 3-sec spacing of shocks in the early and late massed groups might have reduced the discriminability of the brief, unreinforced intershock interval. That 
is, these groups may have perceived the contextual stimulus as continuously reinforced. Additionally, unlike the early and late groups, shocks in the split group could follow a long shock-free period; that is, the split group had a longer and more variable $\mathrm{N}$-length.

In Experiments 3-5, I examined the possible role of partial reinforcement in the slow extinction of freezing after two bouts of massed shock. In the early and late groups of Experiment 1, unreinforced periods occurred before and after a single bout of massed shock. With a single session of conditioning, these unreinforced periods cannot be described as intervening between reinforced presentations of the context; that is, there is no opportunity for partial reinforcement. However, if two sessions of conditioning are conducted, an unreinforced period following the bout of shock on Day 1 would necessarily precede the next bout of snock on Day 2. It is possible, then, that multiple conditioning sessions could act like the split procedure of Experiment 1 and eliminate the massed-shock deficit. In Experiment 3, rats were given multiple conditioning sessions with massed or distributed shocks. This involved the presentation of a single bout of three shocks in each of two conditioning sessions; 90 -sec periods preceded and followed each bout.

\section{Method}

The subjects were 40 naive rats of the same description as those in the previous experiments. Conditioning and testing occurred in the operant chamber. On Day 1, the rats explored the to-be-conditioned context for $300 \mathrm{sec}$. In the next conditioning phase, 10 of the rats were randomly assigned to each cell of the $2 \times 2$ factorial experiment. Factors were intershock interval ( $3 \mathrm{vs} .60 \mathrm{sec}$ ) and number of days of conditioning (1 vs. 2). Three 1-sec, 0.5-mA shocks were presented at the designated interval; each was preceded and followed by a 90 -sec unreinforced period. Freezing was assessed every $5 \mathrm{sec}$ in a single, 300 -sec test session.

\section{Results and Discussion}

Figure 4 shows the mean percentage of samples in which the rats froze. The effects of intershock interval and number of days of conditioning were additive and not interactive. Distributed shock produced higher levels of freezing than massed shock, and more freezing was evident after two sessions of conditioning than after one session of conditioning $[F(3,36)=8.97 ; 60 \mathrm{sec} / 2$ days $>$ $60 \mathrm{sec} / 1$ day $=3 \mathrm{sec} / 2$ days $>3 \mathrm{sec} / 1$ day]. There was no evidence that the multiple conditioning sessions eliminated the massed-shock deficit.

\section{EXPERIMENT 4}

It would not be unreasonable to suggest that waiting times at the beginning and end of a session may not be perceived as part of the context of conditioning. If so, there would be no basis for the partial reinforcement effects in the preceding experiment. In Experiment 4, massed- and distributed-shock groups were given two sessions of conditioning. Some of the rats in the massed and distributed groups received a partial reinforcement pro-

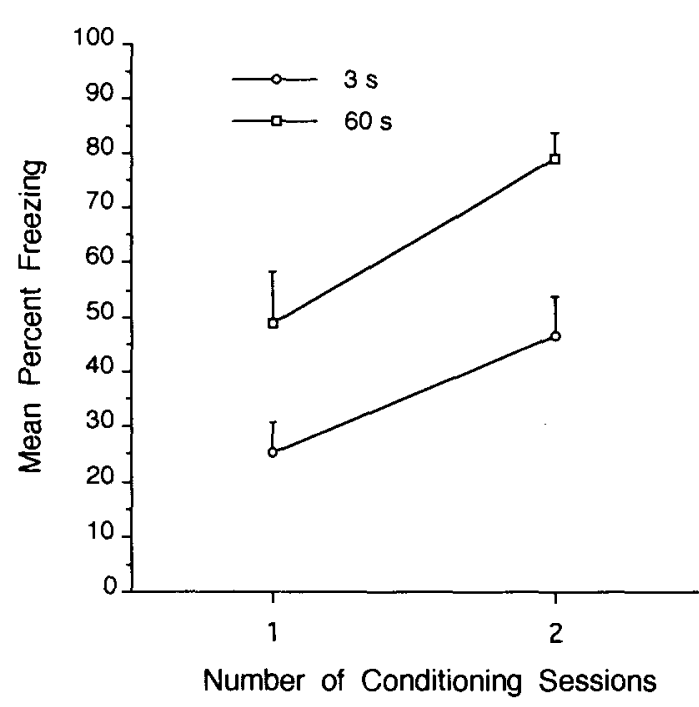

Figure 4. Intershock interval ( 3 vs. 60 sec) and number of days of conditioning (1 vs. 2) have an additive effect on conditioned freezing evoked by a context paired with shock.

cedure in which a session of nonreinforcement intervened between reinforced sessions. Other groups received a continuous reinforcement treatment.

\section{Method}

The subjects were 40 naive rats of the same description as those in the previous experiments. Conditioning and testing occurred in the operant chamber. On Day 1, the rats were preexposed to the conditioning chamber for $300 \mathrm{sec}$. Four groups were then formed by randomly assigning 10 subjects to each cell of the $2 \times 2$ factorial design. During conditioning, bouts of three 0.5 -mA shocks included either distributed (60 sec apart) or massed (3 sec apart) shocks. The first bout of shocks occurred on Day 2 of the experiment, and the second bout occurred on Day 4 . There were 90 -sec waiting periods before the first shock and after the last shock. The partial reinforcement groups were exposed to the conditioning box in the absence of shock on Day 3; this session lasted 300 sec. The continuously reinforced groups remained in their home cages. This conditioning was followed by 5 days of testing. These unreinforced tests lasted $300 \mathrm{sec}$. Freezing was assessed every $5 \mathrm{sec}$ for the entire session.

\section{Results and Discussion}

Experiment 4 provided no evidence that partial reinforcement of context enhanced freezing. Figure 5 shows that extinction of freezing was slower after continuous reinforcement than after partial reinforcement. This outcome was especially evident in the distributed-shock groups. The same relation held in the massed-shock groups, although the numerical differences between the continuous and partial groups were not as marked, because of lower levels of freezing. Reliable simple effects of group were obtained on all 5 days of testing. On Days 1,4 , and 5 , the continuous/60-sec group froze more than the remaining groups, which did not differ from one another $\left[F_{\mathrm{s}}(3,144)\right.$ $\geq 2.22]$. On Day 2 , the two continuous groups froze more than the partial/3-sec group, and the partial $/ 60$-sec 


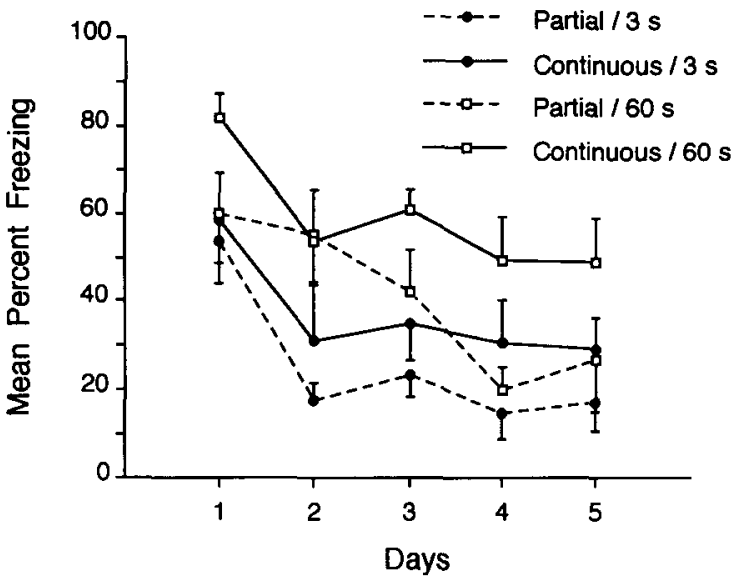

Figure 5. Extinction curves during 5 days of unreinforced freezing tests for groups receiving partial or continuous reinforcement and 3- or 60-sec intershock intervals (Experiment 4).

group was between those extremes $[F(3,144)=4.58]$. On Day 3, the means of the partial/60-sec and continuous/ 3 -sec groups lay intermediately between the means of the continuous/60-sec and partial/3-sec groups $[F(3,144)=$ 3.37]. In summary, the partial/3-sec group froze the leastnot the most-which certainly reduces the attractiveness of the idea that nonreinforcement during the interbout interval of the split procedure of Experiment 1 may have caused the enhanced freezing in that experiment.

\section{EXPERIMENT 5}

In Experiment 4, contextual cues were unreinforced in a separate session. One important feature of a partial reinforcement account of the massed-shock deficit is its prediction that an unreinforced period should precede a bout of reinforcement. Although the combined results of Experiments 3 and 4 rule out between-sessions partial reinforcement effects, they do not rule out within-sessions effects. Within-sessions N-R transitions occurred in the split procedure in Experiment 1, but not in Experiments 3 and 4.

Experiment 5 was modeled after experiments in instrumental learning that have demonstrated within-sessions partial reinforcement effects. Homzie, Rudy, and Carter (1970) reported that penning rats in the goalbox of a runway during the intertrial interval, and not some other place, was an effective means of producing a partial reinforcement effect. In Experiment 5, two groups of rats received a modified split-conditioning procedure. These split groups were treated differently in the interbout interval. One group of rats was removed from the conditioning context and placed in a nearby holding cage (split/ holding). Another group was handled briefly and returned to the conditioning environment during the interbout interval (split/conditioning). A within-sessions partial reinforcement effect would be revealed by greater freezing in the group penned in the conditioning environment. The split/holding group should not freeze at high levels during later testing, because there would be no opportunity for unreinforced periods in the conditioning context to be associated with subsequent reinforcement. Consequently, freezing should rapidly decline in extinction in this group.

\section{Method}

Forty naive, male Sprague-Dawley rats $(250-275 \mathrm{~g})$ were randomly assigned to four conditioning treatments $(n=10)$. The operant chamber was used as the conditioning environment. The closed, white compartment of the choice apparatus, described in Experiment 2 , served as the holding context.

The temporal parameters of shock delivery were based on those in Experiment 1. After exploration of the conditioning context for $730 \mathrm{sec}$ on Day 1, four groups of rats received different conditioning treatments. The critical groups were the split/holding and split/ conditioning groups. Like the split group of Experiment 1 , two bouts of massed shock (five 1-sec, 0.5-mA footshocks, spaced $3 \mathrm{sec}$ apart) were presented in the conditioning environment, separated by an interbout interval of $516 \mathrm{sec}$. The rats in the split/holding group were removed from the conditioning environment during the interbout interval, $90 \mathrm{sec}$ after the termination of Bout 1, and were placed in a nearby holding context. The rats were placed into the white compartment of the choice apparatus and were returned to the conditioning environment $90 \mathrm{sec}$ prior to Bout 2 . The holding context was located on the floor of the room that housed the conditioning context. The room lights, which were extinguished while the rats were in the conditioning context, were illuminated while they were in the holding context. The transfer between contexts required about $5 \mathrm{sec}$. The rats in the split/conditioning group were treated identically, except that, following a brief 5-sec period of handling, they were replaced in the conditioning environment during the interbout interval. Handling occurred on two occasions: $90 \mathrm{sec}$ after termination of Bout 1 and $90 \mathrm{sec}$ prior to Bout 2. The remaining two groups, the distributed and split groups, were direct replications of the corresponding groups in Experiment 1. These groups provided baseline levels of freezing to assess the magnitude of any partial reinforcement effects in the split/conditioning group. Similar to Experiment 1, there were 3 days of unreinforced freezing tests. Freezing was assessed early $(0-90 \mathrm{sec})$, midway $(275-365 \mathrm{sec})$, and late $(550-640 \mathrm{sec})$ in the test sessions.

\section{Results and Discussion}

The mean percentage of samples scored as freezing during the test are shown in Figure 6. The most important finding was that unreinforced exposure to the conditioning context during the interbout interval did not greatly enhance freezing. The rats that had spent the interbout interval in the holding context (split/holding) froze at levels that were similar to those for the rats that were handled and replaced in the conditioning context (split/ conditioning). Together, these two groups froze at much lower levels than the split group, and on two occasions they froze less than the distributed group.

Simple main effects of group were found in six of the nine intervals of the 3-day extinction test $[F \mathrm{~s}(3,101) \geq$ 2.34]. Only the group differences in the middle and late periods of Day 1 and the late period of Day 3 were unreliable. Post hoc tests indicated that the distributed group froze the most in the early interval on Day 1; the remaining groups did not differ from one another. The observation of more freezing in the distributed group than in the split group on initial exposure to the context replicates 


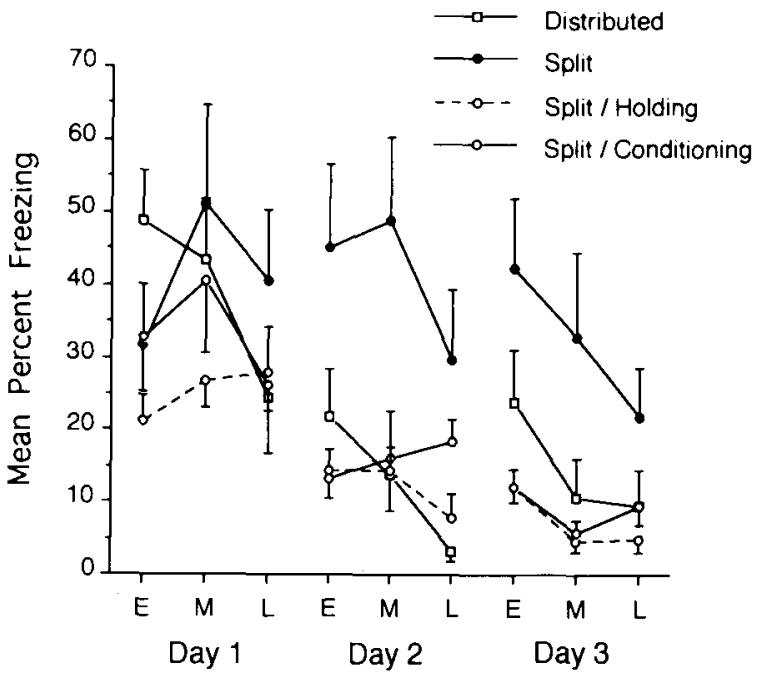

Figure 6. Mean percentage of freezing in Experiment 5 over 3 days of unreinforced testing as a function of group (distributed, split, split/holding, split/conditioning) during the three daily observation intervals $(E=$ early, or $0-90 \mathrm{sec} ; M=$ middle, or $275-365$ sec; and $L=$ late, or 550-640 sec).

the findings of Experiment 1. Similar to that experiment, freezing also declined much more rapidly in the distributed group than in the split group. On Day 2, group orders were reversed, and the split group froze more than the distributed group. Freezing in the distributed group was similar to that in the split/conditioning and split/holding groups in all three intervals. On Day 3, the split group froze more than the split/conditioning and split/holding groups in the early interval, and the distributed group froze at intermediate levels. The split group froze-more than the other three groups in the middle interval of Day 3.

The findings of the present experiment are inconsistent with the view that slow extinction following exposure to two bouts of massed shock is due to partial reinforcement. One could argue that handling disrupted processing of contextual stimuli in the split/conditioning group and interfered with partial reinforcement. Although this is possible, it is not clear why analogous experiments in instrumental learning have revealed partial reinforcement effects, whereas the present experiment did not. Experiment 5 also confirmed that there was a lower level of freezing in the split group than in the distributed group during the early interval of Day 1. This suggests that dividing 10 shocks into early and late bouts had little initial effect, but somehow retarded extinction as the testing continued. This latter effect apparently depends on the rats' being left undisturbed during the interbout interval.

\section{EXPERIMENT 6}

At this point, other interpretations of slow extinction after two bouts of massed shock need to be considered. The results from the preceding experiments are consistent with the view that temporal stimuli acquire control over conditioned responding. The animals in the split group may have learned to time the interbout interval or to localize shock to early and late conditioning times. Such temporal associations may have prolonged freezing and slowed extinction. The temporal conditioning hypothesis also predicts that interbout handling should disrupt timing and eliminate the enhancement of conditioned freezing resulting from two bouts of massed shock (Experiment 5). Also, separate sessions of massed shock should not have had the same effect as did two bouts of shock in a single session (Experiments 3 and 4).

Although the results of the preceding experiments are consistent with the temporal conditioning hypothesis, they also may be explained in yet another way. Unlike the fixed 60-sec intervals used in the distributed procedure, the spacing between shocks was variable in the split procedure (a single 516-sec interval was mixed with eight 3-sec intervals). If the US was viewed as occurring unpredictably in time, the rats may have become chronically afraid and may have frozen throughout the test session.

In Experiment 6, I assessed the effects of unpredictability on conditioned freezing. Two groups of rats were exposed to 10 shocks on a 60 -sec schedule. In the distributed group, the shocks occurred at fixed 60 -sec intervals. The other group was exposed to a progressive schedule of intershock intervals, averaging $60 \mathrm{sec}$, in which the probability of the US remained constant as a function of time measured from the previous US (see Fleshler \& Hoffman, 1962). Thus, this schedule eliminated temporal conditioning as a factor. If unpredictability enhances freezing, the progressive group of Experiment 6 should mimic the split groups of Experiments 1 and 5 and freeze at higher levels than a distributed group. Considering nonassociative factors, one would not expect substantially lower levels of conditioning in the progressive group than in the distributed group. Only two of the nine intershock intervals in the progressive schedule were less than $16 \mathrm{sec}$.

\section{Method}

Eleven male Sprague-Dawley rats (250-300 g) were randomly assigned to distributed and progressive groups. Conditioning and testing occurred in the operant chamber. The distributed group was treated in the same manner as in Experiments 1 and 5. Following a 730 -sec pretest, 10 shocks spaced $60 \mathrm{sec}$ apart were presented during conditioning. The US was a $1-\mathrm{sec}, 0.7-\mathrm{mA}$ scrambled gridshock. The first and last shocks in the progressive group occurred at the same time as those for the distributed group. However, the nine intervals that separated the intervening USs varied in duration $(3.5,11.0,19.6,29.6,41.7,56.9,77.3,108.7$, or $191.8 \mathrm{sec})$. Each rat was exposed to a different random order of the nine intervals. This single day of conditioning was followed by 3 days of extinction testing. These tests are described in Experiments 1 and 5.

\section{Results and Discussion}

Extinction was more gradual in this experiment than in the preceding experiments because of the higher shockintensity level $(0.7 \mathrm{~mA}$ vs. $0.5 \mathrm{~mA})$. The most interesting finding was that the unpredictable shocks did not produce slower extinction of conditioned responding than did the predictable shocks spaced at fixed 60 -sec intervals (see Figure 7). The extinction curves of the progressive and 
distributed groups were similar, with the exception of the first observation interval of Day 1 . The lower level of freezing in the progressive group in the early interval of Day 1 was reliable $[F(1,94)=4.62]$. The groups did not differ over the next eight observation intervals, using the pooled ANOVA error. The numerical difference did not reach significance in the early interval of Day 2.

Although unpredictability cannot account for the splitshock effect, variable intershock intervals could have played a role in the low levels of conditioned freezing that were observed in the split procedure on initial exposure to the test context. In Experiments 1 and 5, the split group froze less than did the distributed group in the first observation interval of the first test session. The same result for the progressive group of Experiment 6 suggests that difficulties in timing the first shock may diminish conditioned freezing. That is, variable intershock intervals may cause retroactive interference in the animal's timing of the first shock. Another possibility is that distributed and progressive schedules have a differential effect on the shock UR. As mentioned previously, Fanselow et al. (1993) have shown that a vigorous UR to shock may interfere with conditionability. Although this sensitization effect does not explain the split-shock effect (see Experiment 2), it might explain the differences between the distributed and progressive groups. Less sensitization might be expected in the progressive group, because two of the nine intervals were clearly within the range of intershock intervals encompassed by the massed-shock deficit. However, this account does not explain the within-sessions effects in Experiment 6. If there was less sensitization in the progressive group than in the distributed group, it should not have produced a freezing deficit that disappeared after the early scoring interval.

\section{GENERAL DISCUSSION}

In the present experiments I explored the mechanisms underlying slow extinction of conditioned responding

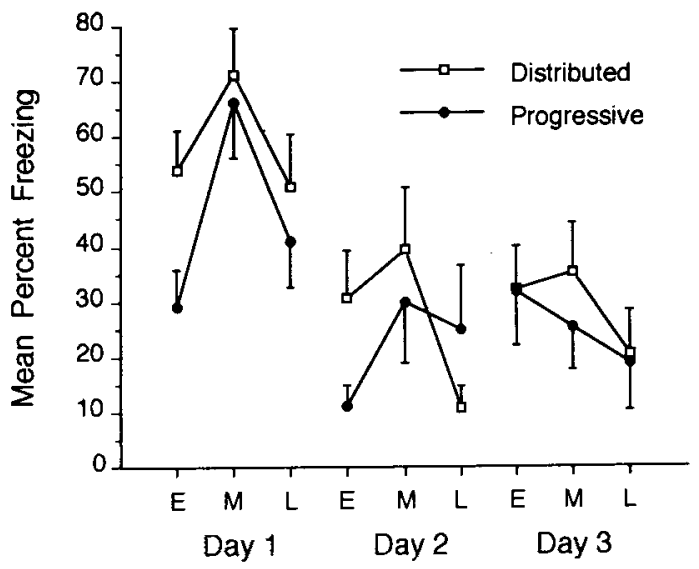

Figure 7. Results of the 3 days of freezing tests in Experiment 6 for the distributed and progressive groups during the three daily observation intervals ( $E=$ early, or 0-90 sec; $M=$ middle, or 275-365 sec; and $L=$ late, or 550-640 sec). when two bouts of presumably ineffective massed shocks were separated by a long interbout interval. In Experiments 1 and 5, extinction of conditioned freezing was slower in a group that received two bouts of massed shock (5 shocks, 3 sec apart) than it was for a group that received the same number of shocks distributed at fixed 60 -sec intervals. Slow extinction of freezing in this split-conditioning procedure was not consistent with an opponent-process mechanism. According to that view, conditioning should have been maximal when the primary affective reaction to the US was not dampened by the unconditional aftereffects of a prior shock. The account provides no explanation for how massed shock could ever act as an effective reinforcer.

Several rival interpretations of slow extinction following two bouts of massed shock were entertained and then abandoned. The first bout of shocks did not have a sensitizing effect on subsequent conditioning produced by the second bout. If anything, the second bout was a less effective reinforcer than was the first bout, as evidenced by transsituational habituation (Experiment 2 ). Thus, preexposure to massed shock does not have the same sensitizing effect as does preexposure to distributed shock (e.g., Fanselow et al., 1993). In Experiments 3-5, I found little evidence that partial reinforcement of a context, either between or within sessions, enhances conditioned freezing. Experiment 6 eliminated unpredictability as a cause of the slow extinction following two bouts of massed shock. A progressive schedule of US presentations did not produce higher levels of freezing than did a standard 60-sec distributed schedule.

There was, however, strong evidence for a role of temporal conditioning in the split-shock effect. One plausible explanation of the data is that bouts of massed USs had acquired signaling properties. In the split procedure, the first bout may have acted as a signal for the second bout. If so, each day of testing may have been perceived by the rats in the split groups as containing only two extinction trials. This bout-to-bout signaling mechanism would retard extinction, compared with the 10 extinction trials that probably were perceived by the rats in the distributed groups. Also consistent with this account is the observation that handling during the interbout interval disrupted freezing in the split/conditioning group in Experiment 5.

A related formulation might propose that the early- and late-session time cues independently acquired associations with the massed-shock US. Summation of moderate levels of temporal conditioning at two points in time could augment freezing. Because freezing is a diffuse, preparatory $\mathrm{CR}$, one would not expect the underlying temporal learning to result in freezing only in the early and late intervals of the session and not during the middle interval. Note that adding extinction curves for the early and late groups in Experiment 1 roughly approximates the curve for the split group.

Whichever of these temporal conditioning accounts is preferred, it is clear that the rats in the split groups learned more than a simple context-US relation. Otherwise, the rate of extinction in the split groups would have been dic- 
tated by the initial level of freezing in the first observation period on Day 1 of extinction. This outcome was not obtained in the present experiments. Additionally, the rats that were given massed shock either early or late in the conditioning session froze in response to localized temporal cues, providing direct evidence of temporal conditioning processes in this paradigm (see also Williams, Frame, \& LoLordo, 1992).

The research reported in the present experiments has general implications for the use of the conditioned freezing paradigm as a measure of contextual conditioning. For example, it could be argued that contextual learning is best measured by examining freezing immediately upon exposure to the conditioning apparatus. These data could be of primary importance, because in the early scoring period there is no differential opportunity for subjects to discriminate the absence of the US. The present results question the "purity" of such measures of contextual conditioning. In Experiment 6, two schedules with the same average 60 -sec intershock interval engendered different levels of conditioned freezing early in the test session. A progressive schedule resulted in less conditioned freezing than a fixed schedule. Although these data could be interpreted in terms of a difference in the strength of the context's association with the US, freezing did not decrease monotonically over the session, as expected in the progressive group. Despite the compelling logic of the assumption that the first few moments of exposure should be the preferred measure of the context's associative value, the data do not support this assumption. Thus, Experiments 1-6 highlight the need to control both shock density and spacing when comparing independent groups in the conditioned freezing preparation.

On the other hand, the failure to identify partial reinforcement effects in Experiments 3-5 is reassuring because it suggests some correspondence between the level of freezing and overall US density. This is a critical new finding for the freezing measure, and it is consistent with the position of some authors (e.g., Kimble, 1961) who suggest that there is a fundamental difference in the processes underlying Pavlovian and instrumental conditioning (e.g., Gonzalez, Longo, \& Bitterman, 1961; Gormezano \& Coleman, 1975). That is, Pavlovian conditioning is not susceptible to partial reinforcement. This further increases one's confidence in using freezing as a model system for understanding the neural basis of Pavlovian conditioning (e.g., Fanselow, 1991; LeDoux, 1990). Recognition that temporal factors may control freezing under some conditions will also allow study of the physiological mechanisms of this form of learning. It would not be surprising to find a role for the hippocampus, with time of shock as the controlling variable.

\section{REFERENCES}

Balsam, P. D., \& Tomie, A. (1985). Context and learning. Hillsdale, NJ: Erlbaum.

Blanchard, D. C., \& Blanchard, R. J. (1969). Crouching as an index of fear. Journal of Comparative \& Physiological Psychology, 67, 370-375.
CAPALDI, E. J, (1964). Effect of N-length, number of different N-lengths, and number of reinforcements on resistance to extinction. Journal of Experimental Psychology, 68, 230-239.

CAPALDI, E. J. (1966). Partial reinforcement: A hypothesis of sequential effects. Psychological Review, 73, 459-479.

CAPALDI, E. J. (1967). Memory and learning: A sequential viewpoint. In W. K. Honig \& P. H. R. James (Eds.), Animal memory (pp. 115154). New York: Academic Press.

FANSELOW, M. S. (1991). The midbrain periaqueductal gray as a coordinator of action in response to fear and anxiety. In A. Depaulis \& R. Bandler (Eds.), The midbrain periaqueductal gray matter: Functional, anatomical, and immunohistochemical organization (pp. 151173). New York: Plenum.

Fanselow, M. S., DeCola, J. P., \& Young, S. L. (1993). Mechanisms responsible for reduced contextual conditioning with massed unconditional stimuli. Journal of Experimental Psychology: Animal Behavior Processes, 19, 121-137.

Fanselow, M. S., \& Tighe, T. J. (1988). Contextual conditioning with massed versus distributed unconditioned stimuli in the absence of explicit conditional stimuli. Journal of Experimental Psychology: Animal Behavior Processes, 14, 187-199.

FLEShleR, M., \& HofFMAN, H. S. (1962). A progression for generating variable interval schedules. Joumal of the Experimental Analysis of Behavior, 5, 529-530.

GoDDARD, M. J., \& JENKINS, H. M. (1988). Blocking of a CS-US association by a US-US association. Journal of Experimental Psychology: Animal Behavior Processes, 14, 177-186.

Gonzalez, R. C., Longo, N., \& Bitterman, M. E. (1961). Classical conditioning in the fish: Exploratory studies of partial reinforcement. Journal of Comparative \& Physiological Psychology, 54, 452-456.

Gormezano, I., \& Coleman, S. R. (1975). Effects of partial reinforcement on conditioning, conditional probabilities, asymptotic performance, and extinction of the rabbit's nictitating membrane response. Pavlovian Journal of Biological Science, 10, 13-22.

HomziE, M. J., Rudy, J. W., \& CARTER, E. N. (1970). Runway performance in rats as a function of goal-box placements and goal-event sequence. Journal of Comparative \& Physiological Psychology, 71, 283-291.

KrmBLE, G. A. (1961). Hilgard and Marquis' conditioning and learning Englewood Cliffs, NJ: Prentice-Hall.

Landeira-Fernandez, J., Fanselow, M. S., \& De Oca, B. M. (1992). Opposite roles of ventral and dorsolateral periaqueductal gray in conditional fear-related defensive behavior. Society for Neuroscience, 18, 1564. (Abstract No. 652.9)

LEDouX, J. E. (1990). Information flow from sensation to emotion: Plasticity in the neural computation of stimulus value. In $\mathrm{M}$. Gabriel \& J. Moore (Eds.), Learning and computational neuroscience: Foundations of adaptive networks (pp. 3-52). Cambridge, MA: MIT Press.

RoDGER, R. S. (1974). Multiple contrasts, factors, error rate and power. British Journal of Mathematical \& Statistical Psychology, 27, 179-198.

RODGER, R. S. (1975). The number of non-zero, post hoc contrasts from ANOVA and error-rate. British Journal of Mathematical \& Statistical Psychology, 28, 71-78.

Satterthwaite, F. E. (1946). An approximate distribution of the estimates of variance components. Biometrics Bulletin, 2, 110-114.

Solomon, R. L., \& CoRBit, J. D. (1974). An opponent-process theory of motivation: I. The temporal dynamics of affect. Psychological Review, 81, 119-145.

Williams, D. A., Frame, K. A., \& LoLordo, V. M. (1991). Reexamination of contextual conditioning with massed versus distributed unconditioned stimuli. Journal of Experimental Psychology: Animal Behavior Processes, 17, 202-209.

Williams, D. A., Frame, K. A., \& LoLordo, V. M. (1992). Discrete signals for the unconditioned stimulus fail to overshadow contextual or temporal conditioning. Journal of Experimental Psychology: Animal Behavior Processes, 18, 41-55.

(Manuscript received March 19, 1993; revision accepted for publication October $7,1993$. 